This item was submitted to Loughborough's Research Repository by the author.

Items in Figshare are protected by copyright, with all rights reserved, unless otherwise indicated.

\title{
Automotive wheel and tyre design for suppression of acoustic cavity noise through the incorporation of passive resonators
}

\section{PLEASE CITE THE PUBLISHED VERSION}

https://doi.org/10.1016/j.jsv.2019.115037

\section{PUBLISHER}

Elsevier

\section{VERSION}

AM (Accepted Manuscript)

\section{PUBLISHER STATEMENT}

This paper was accepted for publication in the journal Journal of Sound and Vibration and the definitive published version is available at https://doi.org/10.1016/j.jsv.2019.115037.

\section{LICENCE}

CC BY-NC-ND 4.0

\section{REPOSITORY RECORD}

O'Boy, Daniel. 2019. "Automotive Wheel and Tyre Design for Suppression of Acoustic Cavity Noise Through the Incorporation of Passive Resonators". Loughborough University. https://hdl.handle.net/2134/9918527.v1. 
1

2

3

\section{Automotive wheel and tyre design for suppression of acoustic cavity noise through the incorporation of passive resonators}

\author{
Daniel J. O'Boy \\ Department of Aeronautical and Automotive Engineering, Loughborough University, \\ Loughborough, Leicestershire LE11 3TU, United Kingdom.
}

\begin{abstract}
Tyre cavity noise is a narrow band, high amplitude resonance which occurs when the sound waves in a car tyre oscillate back and forth in one direction with a low loss factor. Manufacturers add damping materials to vehicles, due to the difficulty of reducing the source noise, or mitigating the transmission path from wheel hub to the interior, as part of reducing the overall structure borne noise.

When the wheel is rolling, the Doppler effect leads to two characteristic cavity resonance frequencies, which change with speed, loading and temperature. If these couple with a suspension component resonance, a transmission path to the cabin can result. One method to attenuate the sound is by using passive resonators, such as Helmholtz and quarter wavelength resonators. Here, a rapid design method is developed, based around one-dimensional waveguide equations in order to optimise the dimensions and thus the tuned resonant frequency of the wheel based Helmholtz resonators.

With three tuned resonators, a reduction in the peak sound amplitude from 103 to $88 \mathrm{~dB}$ is possible, over a speed range from $54 \mathrm{~km} / \mathrm{hr}$ to $108 \mathrm{~km} / \mathrm{hr}$. With five resonators, a reduction from 103 to approximately $87 \mathrm{~dB}$ is shown. Thus, the larger number of resonators is better able to attenuate the cavity noise over a larger frequency range.

Keywords: tyre, noise, automotive, wheel, resonator, Helmholtz, cavity resonance
\end{abstract}

Email address: d.j.oboy@lboro.ac.uk (Daniel J. O'Boy) 


\section{Introduction}

The emphasis on a quiet interior cannot be understated in a competitive automobile design market. Traditional noise, vibration and harshness (NVH) optimisation techniques focus on the most significant noise sources, such as

5 engine, drivetrain and exhaust sounds, in addition to understanding the common transmission paths 1, 2]. For example, Sakata et al. state that the interior noise below $300 \mathrm{~Hz}$ is dominated by transmission through structural members [3]. Some of these sounds are accentuated or tuned to the customer expectation for a given vehicle type while others are masked by these dominant sources,

10 including components rubbing, rattles, squeaks and electric motor whirr.

The introduction of electric and hybrid vehicles means the engine no longer produces a high amplitude, broad-band noise that can be assumed to mask these other sounds; an advantage for the consumer enjoying a quiet interior and a challenge for the designer. One problematic sound is the acoustic resonance that can occur within the tyre itself, termed "tyre cavity resonance". Without the engine noise this is a highly noticeable sound that can require the introduction of additional damping material to mitigate the effects of the transmission path, with an associated increase of carbon dioxide costs, manufacturing and installation complexity, a reduction in fuel efficiency and in-vehicle space.

The problem of tyre cavity resonance is understood within the literature as even for combustion engine vehicles it is important for NVH optimisation. Addressing this noise is carried out by the determination of the tyre cavity natural frequency (either experimentally or numerically [4]) and then the appropriate design of wheel and suspension components are chosen in order to minimise the noise transmission path.

The NVH issues associated with electric and hybrid vehicles are different to those with petrochemical detonation engines, as squeaks, rattles and mechanical clunks become the primary concern, characterised as transient noise sources. The tyre cavity noise is particularly problematic in this case as it usually occurs in a narrow frequency range around $170-250 \mathrm{~Hz}$ where it is noticeable and difficult to mitigate in the vehicle design stage as the natural frequency is dependent on the wheel shape and size [5], which may not be finalised.

In this paper, a design methodology for addressing tyre cavity noise will be 
presented by using a combination of passive resonators to suppress the cavity 5 resonance and hence to prevent the sound reaching the cabin.

While active techniques provide a significant reduction over a wider frequency range, they are expensive, potentially prone to failure and require more space. Prediction tools for estimating the tyre cavity natural frequency vary from accurate (and potentially time consuming, depending on the fidelity, resolution) finite element [6], boundary element models (where the tyre geometry is taken into account) to fast, simplified waveguide models where the tyre sidewall curvature is neglected [4]. This type of waveguide model is implemented in this paper in order to examine the tyre cavity acoustic natural frequencies, notably by inclusion of the effects of rotation of the wheel / tyre, a loaded contact patch and temperature variations. However, the inclusion of individually tuned passive Helmholtz resonators are detailed in order to attenuate the cavity resonances over a wide frequency range, which is a novel addition to the existing methods. As individual passive resonators have a relatively narrow operating bandwidth 7], design optimisation on an unloaded and non-rotating tyre will ${ }_{50}$ produce an inferior result in practise, as the tyre cavity resonances alter with a number of variables [5, 8, 4, 9]. By taking these operating conditions into account in this paper, a specimen tyre and wheel with low cavity noise can be obtained, which is an important addition to knowledge in the literature.

A background is briefly described in section 1.1 while section 2 contains a 55 description of the main subject of this paper, the waveguide model, followed by predicted results and discussion pertinent to tyre rotation speed and contact patch length. The methodology for including the resonators is shown in section 3 and conclusions given where important design factors are listed. The overall aim is to demonstrate robust design techniques for the prediction of sound attenuation from passive resonators on loaded, rotating automotive tyres. The method in this paper means that an industrial design engineer can quickly find i) the sound pressure levels in the tyre, while in operating conditions and ii) determine the optimum resonator designs to mitigate this level, all for operating conditions.

The transmission path to the driver and passenger is complex and bespoke to each vehicle, hence the coupling between the air cavity and tyre, and tyre 
Figure 1: Cross-section of a generic wheel and tyre showing the cavity volume bounded by the wheel and tyre materials.

to vehicle interior is not considered. This then allows the vehicle designer to address the noise at source without concern for trying to minimise amplitudes of sound and vibration at frequencies relating to suspension design. One key 70 advantage to the method in this paper is that the simplified waveguide model allows the noise to be reduced at source using resonators with clear physical insight, whereas to take into account the tyre construction, suspension linkage, vehicle trim and interior noise would likely need a supercomputer running FEM models and knowledge of all geometrical, material and loss factors on that transmission path.

\subsection{Tyre cavity noise, a physical interpretation}

The air inside the tyre, bounded by the inner tread rubber, sidewalls and metal wheel is a lightly damped fluid in a cylindrical annulus shape, as shown in the wheel / tyre cross-section in Fig. 1. The air column is excited by movement of the tread rubber at the tyre contact patch over a broadband frequency range, leading to a number of cavity resonances being generated where the air mass moves independently of the tyre width.

The geometry may be represented by a cylindrical annulus, where the pressure perturbation varies with radius and circumferential position. The pressure 85 perturbation inside the cavity is given by $p^{\prime}$ and the speed of sound $c$. The wave equation governing sound propagation is given in cylindrical coordinates (radius $r$, circumferential position $\theta$ ) [10] $\frac{\partial^{2} p^{\prime}}{\partial r^{2}}+\frac{1}{r} \frac{\partial p^{\prime}}{\partial r}+\frac{1}{r^{2}} \frac{\partial^{2} p^{\prime}}{\partial \theta^{2}}=\frac{1}{c^{2}} \frac{\partial^{2} p^{\prime}}{\partial t^{2}}$. It is assumed that the pressure perturbation is invariant in the axial direction, such that it only varies with radius, time and angular position through a harmonic 
function, $p^{\prime}(r, \theta, t)=\frac{1}{(2 \pi)^{2}} \int_{-\infty}^{\infty} \sum_{n=-\infty}^{\infty} \tilde{p}(r, n, \omega) e^{\mathrm{i} n \theta} e^{\mathrm{i} \omega t} d \omega$. As a wave travelling around the cavity in one direction must meet with one travelling in the opposite direction, due to periodicity, the angular order $n$ is an integer mode number $(0,1,2, \ldots)$ (the pressure variation with mode is described in O'Boy et al. [11]).

The mode $n=0$ is a pulsating mode while $n=1$ corresponds to the air column moving up and down, which yields the only net time-varying force on the wheel hub, thus leading to the structure-borne vibration and sound in the passenger cabin (the tyre cavity mode). For the case of a tyre that is not rotating, the first mode is degenerate, therefore, it may appear as a valid solution rotated by ninety degrees (also called the free-free case), however, once the tyre is spinning, there is a separation of frequencies due to the unequal travel length of the acoustic waves.

A simple estimate of the natural frequency of the cavity can be found through a solution of the wave equation using $\Gamma=\omega / c$ to obtain the radial pressure distribution in terms of Bessel's functions, thus $\tilde{p}^{\prime}(r)=c_{1} J_{n}(\Gamma r)+c_{2} Y_{n}(\Gamma r)$. The constants $c_{1}, c_{2}$ are found through the implementation of appropriate boundary conditions on the outer and inner radial surfaces (denoted $R_{o}$ and $R_{i}$ ). For example, zero fluid velocity, $\tilde{u}_{r}=\frac{1}{\rho_{o}(\mathrm{i} \omega)} \frac{\partial \tilde{p}^{\prime}}{\partial r}$, leads to a first natural frequency for the $n=1$ mode of approximately $180-250 \mathrm{~Hz}$ for commercial tyre and wheel dimensions (where the mean density of air is $\rho_{0}$ and the speed of sound $c_{0}$ ).

When the tyre diameter is sufficiently large, this first cylindrical acoustic mode may be sufficiently represented by the first even mode in Cartesian coordinates [4, 3], the basis of the waveguide model used in this paper. Given a total length of waveguide of $2 \pi R_{\text {eff }}$, where $R_{\text {eff }}$ is the effective radius of the tyre, and a set of waves propagating in both directions of the form $p^{\prime}(x, t)=\frac{1}{(2 \pi)^{2}} \int_{-\infty}^{\infty} \int_{-\infty}^{\infty} \tilde{p}\left(k_{x}, \omega\right) e^{\mathrm{i} k_{x} x} e^{\mathrm{i} \omega t} d k_{x} d \omega$, with periodic boundary conditions at the ends of the waveguide (a Cartesian model equivalent to the cylindrical model can be constructed). The equivalent mode for the Cartesian system is $k_{x}=m \pi / 2 \pi R_{\text {eff, }}$ where $m=2$ (the $m=2$ mode in the Cartesian coordinates model is then directly comparable with the $n=1$ mode in the cylindrical coordinates model). In this case the effective radius $R_{\mathrm{eff}}=\left(R_{o}+R_{i}\right) / 2$ (the inner radius is given by the hub radius, while the outer radius is estimated using the 


\begin{tabular}{c|cc|c}
\hline \hline Tyre size & Cart. modes [Hz] & Cyl. modes [Hz] & Difference (\%) \\
\hline 175/70R14 & 228.36 & 230.67 & -1.00 \\
165/60R14 & 240.17 & 241.96 & -0.74 \\
195/70R15 & 210.98 & 213.22 & -1.05 \\
195/50R15 & 228.17 & 229.68 & -0.66 \\
205/55R16 & 210.31 & 211.87 & -0.74 \\
$215 / 65 \mathrm{R} 16$ & 199.91 & 201.92 & -1.00 \\
195/45R16 & 220.95 & 222.07 & -0.50 \\
$235 / 65 \mathrm{R} 17$ & 186.78 & 188.73 & -1.04 \\
205/40R17 & 212.50 & 213.37 & -0.41 \\
$235 / 60 \mathrm{R} 18$ & 182.51 & 184.10 & -0.86 \\
225/40R18 & 199.53 & 200.40 & -0.44 \\
255/35R19 & 190.92 & 191.68 & -0.39 \\
\hline \hline
\end{tabular}

Table 1: Frequency of the first tyre cavity mode [Hz] for a range of tyres when calculated using the cylindrical and Cartesian models and the percentage difference between the predicted values.

overall tyre diameter (the thickness of rubber is assumed more or less equal to the change in radius of the wheel rim seen in Fig. 10). A comparison of the accuracy of these two methods is shown in Table 1 where the fundamental tyre cavity mode is estimated using both the cylindrical and Cartesian model approximations for a range of commonly found tyre sizes on different rims. Table 1 shows that changing the wheel size changes the fundamental frequencies and thus can quite easily interrupt the noise transmission path. Conversely, inappropriately changing the wheel size can quite easily couple natural frequencies and create a transmission path to the cabin. Table 1 also shows that the Cartesian waveguide model produced in the results section consistently underestimates the natural frequency predicted by the cylindrical model by approximately one percent, but this is still useful for initial design purposes. See also Thompson [4] for examples of predicted cavity natural frequencies against experimentally measured frequencies.

\subsection{General understanding of tyre cavity noise and best practice}

The wheel environment is harsh and the incorporation of sound mitigation devices has not become mainstream, nor have active resonators showed use, although the designs in this paper are applicable, providing any geometry changes are slow.

Measurements on the spindle of a rolling wheel by Sakata et al. 3] show 
a strong vibration amplitude at three frequencies which could be transmitted to the vehicle interior, identified as structural resonances of the tyre (circumresonance from predictions based on a periodic representation of a waveguide in Cartesian coordinates, used as the basis for the development of the more advanced model presented in this paper. It was shown [3] that the force vectors from the second and third acoustic modes cancel to produce no net force on the hub and spindle, while the first mode does yield a net force, which is why it is the only one to be transmitted through to the cabin. A finite element model was also generated to confirm the findings that the resonant frequency does not change with inflation pressure or a change in carcass stiffness (as this does not affect the speed of sound and so can be decoupled).

It is presumed in this paper that for the purposes of calculating the acoustic cavity mode, the acoustic cavity model can be decoupled from the structural tyre model and used in isolation to examine designs that reduce the transmitted force to the wheel hub. One reason the acoustic model can be assumed to be decoupled is that the high damping in the tyre materials does not appear to attenuate the acoustic cavity mode [6], nor does the tyre structure alter the cavity resonance. However, at the tyre cavity resonance frequency, the coupling of the acoustic cavity and structural tyre carcass vibrations means that the tyre vibration is greatly increased (not concerning this paper). Kim et al. 12, 13] use finite element models which demonstrate this coupled behaviour, deriving sound radiation predictions from each part of the tyre, including sidewall, tread compound and tread variation with position. Leaving the design tool uncoupled from the structural response is not as accurate as a fully coupled model, however it means that the vehicle designer need not be concerned with the determination of the individual layers of a tyre carcass, the response of the non-linear rubber to temperature and strain parameters and the inflation / load responses of the rubber materials. See O'Boy and Dowling [11] for examples of tyre belt modelling.

The amplitude of the transmitted interior sound depends on the direct and indirect transmission paths, for example, whether the wheel and hub have natural frequencies near to the tyre cavity natural frequency. Scavuzzo et al. have 
shown that when a wheel bending mode coincides with the cavity resonant frequency, the sound pressure inside the vehicle is $78 \mathrm{~dB}$ when fitted with a steel wheel, in contrast to $66 \mathrm{~dB}$ when an aluminium wheel with a far different wheel bending frequency is chosen [5]. Similarly the use of Helium decreases the transmitted sound from both wheel types to within $54-56 \mathrm{~dB}$, as the speed of sound of Helium is far greater [5] than air. Feng and Gu suggest that a current design guideline whereby modal frequencies are separated by $20-30 \mathrm{~Hz}$ does not produce a robust low vibration and noise transmission path, instead some resonances can appear amplified [14].

\section{A tyre cavity waveguide model with tyre rotation and loading}

The numerical method uses Cartesian co-ordinates with continuous boundary conditions on either end of the waveguide to emulate an annular geometry. Each waveguide section has a constant thickness, width and speed of sound. With reference to Fig. 2, each individual section is denoted by the index $j$. ${ }_{190}$ Thus the circumferential position $x_{j}$ can vary between 0 and $2 \pi R_{\text {eff }}$, at which point periodicity applies.

The maximum frequency is dictated by the cut-on frequency for higher order radial (or axial) modes of the tyre cavity, but a coincidental advantage is that for interior noise propagation from the tyre into the cabin, an upper limit of approximately $500 \mathrm{~Hz}$ is typically considered for NVH analyses.

\subsection{Waveguide sections and boundary conditions}

Assume the position of the end of section $j$ is the same at the start of section $j+1$, thus $x_{j}=x_{j+1}$. Then the pressure perturbation $p^{\prime}\left(x_{j}\right)$ and mass flux $\dot{m}\left(x_{j}\right)$ at the start of the section can be arranged in vector form as $\mathbf{Y}_{x_{j}}=\left[p^{\prime}\left(x_{j}\right), \dot{m}\left(x_{j}\right)\right]^{\mathrm{T}}$, where

$$
\mathbf{Y}_{x_{j}}=\left[\begin{array}{cc}
1 & 1 \\
\frac{A}{c_{j}} & -\frac{A}{c_{j}}
\end{array}\right]\left[\begin{array}{cc}
e^{\mathrm{i} \omega\left(t-\frac{x_{j}}{c_{j}}\right)} & 0 \\
0 & e^{\mathrm{i} \omega\left(t+\frac{x_{j}}{c_{j}}\right)}
\end{array}\right]\left[\begin{array}{c}
c_{1} \\
c_{2}
\end{array}\right] .
$$

The complex constants $c_{1}$ and $c_{2}$ now represent the amplitudes of the acoustic waves travelling in the right and left directions, respectively, a method used in simplified form by Thompson [4]. The cross-section area of this section is $A$ 
$(a)$

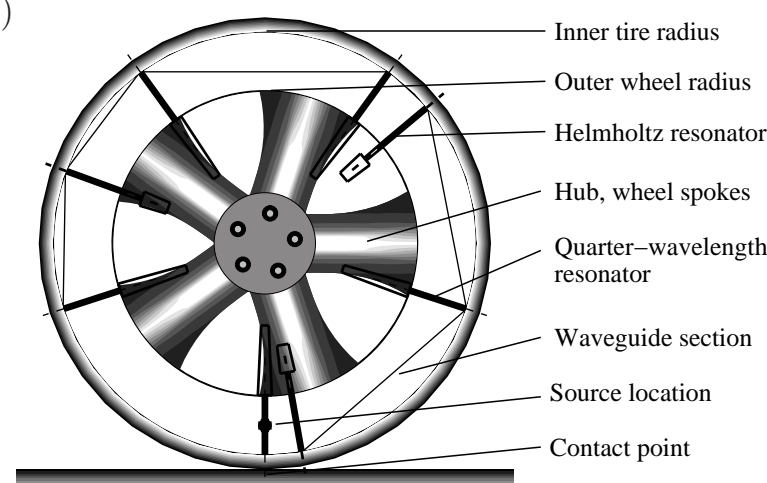

(b)

\begin{tabular}{|c|c|c|c|}
\hline \multicolumn{4}{|c|}{ Waveguide boundaries } \\
\hline Section $j-1$ & & $n j$ & Section $j+1$ \\
\hline $\begin{array}{l}c_{j-1} \\
A_{j-1}\end{array}$ & & & $\begin{array}{l}c_{j+1} \\
A_{j+1}\end{array}$ \\
\hline$\rho_{j-1}$ & & & $\rho_{j+1}$ \\
\hline$p^{\prime}\left(j-1, x_{j}\right)$ & $p^{\prime}\left(j, x_{j}\right)$ & $p^{\prime}\left(j, x_{j+1}\right)$ & $p^{\prime}\left(j+1, x_{j+1}\right)$ \\
\hline$\dot{m}\left(j-1, x_{j}\right)$ & $\dot{m}\left(j, x_{j}\right)$ & $\dot{m}\left(j, x_{j+1}\right)$ & $\dot{m}\left(j+1, x_{j+1}\right)$ \\
\hline
\end{tabular}

Figure 2: (a) Rectangular Cartesian waveguide model for the tyre cavity geometry, drawn in the equivalent cylindrical reference frame, (b) notation for an individual section in the waveguide.

where the width of the section may change when tyre loading is incorporated. The pressure and mass flux at the far end of the waveguide section can be written using similar notation where the matrix notation $\boldsymbol{\alpha}$ and $\boldsymbol{\Gamma}$ are introduced. As the waveguide is isotropic and homogeneous, the constants are identical to those in Eq. 1 thus,

$$
\mathbf{Y}_{x_{j+1}}=\boldsymbol{\alpha}_{x_{j+1}} \quad \boldsymbol{\Gamma}_{x_{j+1}}\left[\begin{array}{c}
c_{1} \\
c_{2}
\end{array}\right] .
$$

Through simple inversion, the pressure and mass flux on one side of the section can be related to those on the other by,

$$
\mathbf{Y}_{x_{j}}=\boldsymbol{\alpha}_{x_{j}} \boldsymbol{\Gamma}_{x_{j}} \quad \boldsymbol{\Gamma}_{x_{j+1}}^{-1} \boldsymbol{\alpha}_{x_{j+1}}^{-1} \quad \mathbf{Y}_{x_{j+1}} .
$$

This product of four matrices can be summarised in matrix notation as $\boldsymbol{W}_{x_{j}}$ and represents the transfer function for this one section of the waveguide, linking 
the physical parameters on one side to the other. Thus,

$$
\mathbf{W}_{x_{j}}=\boldsymbol{\alpha}_{x_{j}} \quad \boldsymbol{\Gamma}_{x_{j}} \quad \boldsymbol{\Gamma}_{x_{j+1}}^{-1} \boldsymbol{\alpha}_{x_{j+1}}^{-1}
$$

In order to complete the waveguide model of the whole tyre cavity, multiple sections need to be joined together as shown in Fig. 2. It is assumed that at the join between neighbouring faces, there is a continuity of pressure and mass flux, which then allows a transfer function to be produced linking the properties on one end of the waveguide to the other, through the entire $N$ sections, thus,

$$
\mathbf{Y}_{x=0}=\prod_{j=1}^{j=N} \quad \mathbf{W}_{x_{j}} \quad \mathbf{Y}_{x=2 \pi R_{\mathrm{eff}}}
$$

\subsection{Forced acoustic resonances}

A source term is required in order to obtain the forced harmonic pressure response, applied as a radial perturbation on the equivalent position of the tyre carcass as $p^{\prime}(x, y, t)=\frac{1}{(2 \pi)^{2}} \int_{-\infty}^{\infty} \int_{-\infty}^{\infty} f\left(y, k_{x}, \omega\right) e^{\mathrm{i} k_{x} x} e^{\mathrm{i} \omega t} d k_{x} d \omega$, where the waves in the radial direction are represented in Cartesian form as $f(y)=$ $\frac{1}{2 \pi} \int_{-\infty}^{\infty} I_{1} e^{\mathrm{i} \gamma y}+I_{2} e^{-\mathrm{i} \gamma y} d \gamma$ where $\gamma^{2}=\omega^{2} / c^{2}-k_{x}{ }^{2}$, illustrated in Fig. 3. On the inner tyre radial surface, $y=R_{i}$, the mass flux is zero, which eliminates one of the constants. On the outer radial surface $y=R_{o}$, the excitation can be written,

$$
p^{\prime}\left(x=0, R_{o}, \omega\right) \propto \frac{1}{(2 \pi)^{2}} \int_{-\infty}^{\infty} \int_{-\infty}^{\infty} \frac{e^{\mathrm{i} \gamma R_{o}}+e^{2 \mathrm{i} \gamma R_{i}} e^{-\mathrm{i} \gamma R_{o}}}{e^{\mathrm{i} \gamma R_{o}}-e^{2 \mathrm{i} \gamma R_{i}} e^{-\mathrm{i} \gamma R_{o}}} e^{\mathrm{i} \gamma R_{o}} d \gamma d k_{x}
$$

A numerical routine determines every value of $k_{x}$ for which the initial pressure term $p_{0}(x=0)=p_{0}\left(x=2 \pi R_{\text {eff }}\right)$ can be applied as a boundary condition

to the near and far ends of the waveguide, to create the periodicity needed to represent the pseudo-cylindrical tyre cavity. The proportional term in Eq. 6 is obtained through a calibration process described below which ensures that the peak sound pressure level at resonance matches the amplitude found experimentally in the literature by authors inserting microphones into wheel cavity spaces.

Once the pressure perturbation is determined at the same start and end location of the waveguide, it provides two known boundary conditions for use 
Figure 3: Illustration of the initial sound pressure in the waveguide through an initial forcing in the out-of-waveguide plane.

in Eq. 5 to allow the two unknown parameters to be obtained using a simple numerical method. Now that the pressure and mass flux are known at the ends of Eq. [5] it is possible to iteratively move through each of the matrices to find the pressure and mass flux at any "circumferential" position in the waveguide.

Bennetts and Crookes showed that the sound pressure level inside the cavity volume is between $114-117 \mathrm{~dB}$ re $2 \times 10^{-5} \mathrm{~Pa}[15]$, hence the initial pressure condition at the contact patch is set so the maximum pressure at resonance is approximately $110 \mathrm{~dB}$ (this is the proportional term in Eq. 6). Lee et al. show a peak sound pressure amplitude per input force of approximately $115 \mathrm{~dB}$ re $2 \times 10^{-5} \mathrm{~Pa} / \mathrm{N}$ [16] from a broad band noise floor of approximately $80 \mathrm{~dB}$ re $2 \times 10^{-5} \mathrm{~Pa} / \mathrm{N}$, which assists in validating this paper.

Results are based on a 205/55R16 tyre inflated with dry air to a gauge 220 pressure of $30 \mathrm{psi}$ at $68^{\circ} \mathrm{F}$, leading to an outer and inner cavity radius of approximately $316 \mathrm{~mm}$ and $200 \mathrm{~mm}$, respectively, allowing for tread depth and rim height adjustments. For a tyre which is non-rotating and just out of ground contact (no carcass deformation), the sound pressure level inside the cavity is plotted against excitation frequency in Fig. 4, where the primary cavity resonance is located at $211 \mathrm{~Hz}$ with an amplitude of $110 \mathrm{~dB}$ and a characteristically low loss factor (the air has a complex wavenumber representing losses with $\eta \approx 2 \times 10^{-4}[17$ ]). This resonant frequency corresponds to the degenerate mode with two solutions of the same frequency value, which can be separated by ninety degrees in phase (discussed in the literature as horizontal / vertical modes).

Using the method described above to find the pressure and mass flux at any point in the waveguide, it is then possible to search and find the peak sound pressure level in the cavity. Through running the program many times, for different speeds, temperatures or axle weights, it is possible to generate contour 


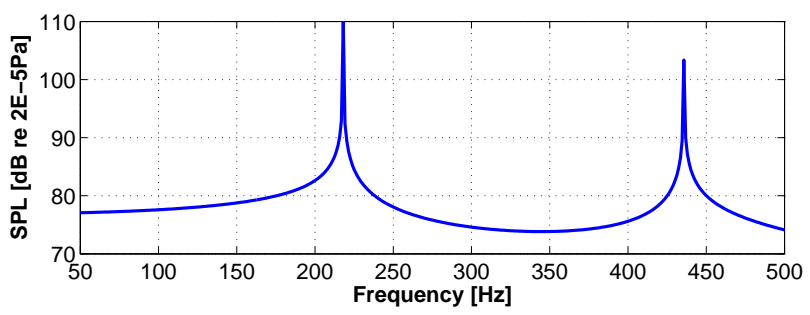

Figure 4: Predicted sound pressure level $[\mathrm{dB}]$ in the tyre cavity for non-rotating wheel, just in contact with the ground.

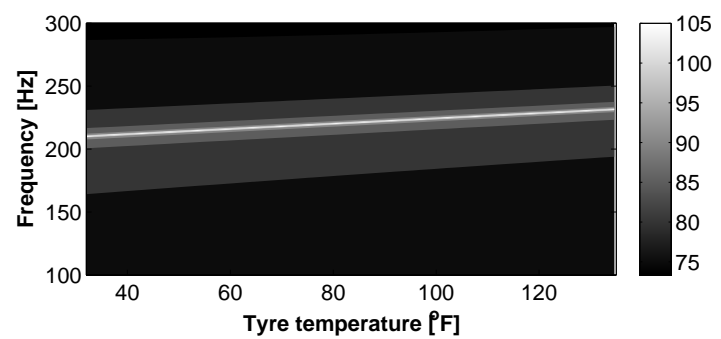

Figure 5: Sound pressure level $[\mathrm{dB}]$ change with respect to tyre temperature and frequency in the tyre cavity for a non-rotating wheel, just in contact with the ground with a uniform increase in gas temperature.

plots of peak sound pressure level as graphs. These are shown in the following 235 results sections.

\subsection{Variation of cavity resonance frequency with temperature and gas}

High performance vehicles can have tyres inflated with nitrogen, carbon dioxide or helium in order to provide certainty over the inflation pressure. Due to friction between tread and road, the speed of sound $c$ varies with absolute temperature $T$ and thus has a marked effect on the tyre cavity resonant frequency, where the lower the temperature, the lower the resonance frequency. This result is illustrated graphically for a non-rotating tyre just out of contact with the ground in Fig. [5] although this change is less significant than seen with a change of vehicle speed. See experiments by Kindt et al. 18] and by Feng et al. 19] for example, where the change in temperature can also be seen to alter the loss factor of the structural modes. The change in cavity resonance frequency with

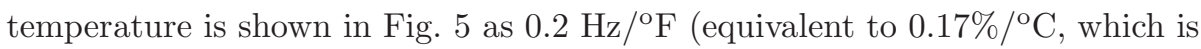
comparable to Kindt's experimental finding of $0.16 \% /{ }^{\circ} \mathrm{C}[18]$ ). 


\begin{tabular}{l|ccc|l}
\hline \hline Gas & $\begin{array}{c}\text { Adiabatic } \\
\text { index, } \gamma\end{array}$ & $\begin{array}{c}\text { Specific gas } \\
\text { constant, } R\end{array}$ & $\begin{array}{c}\text { Density, } \\
\rho_{0}\end{array}$ & $\begin{array}{l}\text { Cavity } \\
\text { freq. }\end{array}$ \\
\hline & & $\mathrm{J} / \mathrm{kg} / \mathrm{K}$ & $\mathrm{kg} / \mathrm{m}^{3}$ & $\mathrm{~Hz}$ \\
\hline Dry air & 1.40 & 287.0 & 1.205 & 211.7 \\
Helium & 1.66 & 2077.5 & 0.166 & 620.1 \\
$\mathrm{CO}_{2}$ & 1.31 & 188.9 & 1.842 & 166.1 \\
Nitrogen & 1.40 & 296.7 & 1.165 & 215.2 \\
\hline \hline
\end{tabular}

Table 2: Tyre cavity natural frequency for a non-rotating tyre, just in ground contact and filled with different gases to a gauge pressure of $30 \mathrm{psi}$ at a temperature of $68^{\circ} \mathrm{F}$.

In the design process, the tyre cavity resonance frequency can be decoupled from the suspension transmission path through the use of a different inflation gas and thus identified [9], as shown in Table 2, where Helium increases the tyre cavity frequency well beyond the range normally considered for interior structure-borne vibration, while carbon dioxide is more readily available.

\subsection{Wheel rotation and tyre loading}

Effective wheel rotation is carried out through manipulation of the speed of sound in each section, assuming that as the tyre rotates with a steady angular velocity, that simultaneously the air in the cavity rotates with no slip between tyre surface and air, which is equivalent to a bias velocity $v$ inside the waveguide, moving at a speed close to that of the tread relative to the hub centre.

From a position fixed to the contact point, there is a Doppler shift in the speed of sound $c_{0}$ propagating in the tyre cavity, where the forward circumferential wave travels at a slower speed $c_{F}=c_{0}-v$ than the speed $c_{B}=c_{0}+v$ of the backward travelling wave. These alterations are incorporated into Eq. [1 for each section of the waveguide. Fernandez provides an alternative formulation where the tread thickness is taken into account [20], including acceleration rather than just steady state analysis.

For zero rotation, acoustic waves travel from the source at the contact patch to the top of the tyre with the same effective length regardless of the direction of travel (clockwise or anticlockwise in Fig. 2). For a rotating tyre, the effective length of travel is altered, leading to two different tyre cavity frequencies as demonstrated in Fig. [6 for a tyre just in contact with the ground at a range of different speeds. If the length of the waveguide is $L=2 \pi R_{\text {eff }}$, then the two 
Figure 6: Sound pressure level $[\mathrm{dB}]$ change with respect to vehicle speed and frequency in the tyre cavity for a rotating wheel, just in contact with the ground.

frequencies are given by Eq. 7, where for the tyre in this paper, the approximate change of cavity frequency with vehicle speed is $0.51 \mathrm{~Hz}$ per $\mathrm{m} / \mathrm{s}$ for the upper solution (equivalent to $1.4 \mathrm{~Hz}$ per $10 \mathrm{~km} / \mathrm{hr}$ ), either by visual inspection from Fig. [ or by differentiation of Eq. 7

$$
F_{1}[\mathrm{~Hz}]=\left(c_{0}+v\right) / L, \quad F_{2}[\mathrm{~Hz}]=\left(c_{0}-v\right) / L
$$

The change in cavity resonance frequency with vehicle speed shown by the contours of Fig. 6 demonstrates the usual problem with designing suspension components to avoid coupling resonances; the cavity frequency can alter by $100 \mathrm{~Hz}$ with normal changes in the vehicle speed.

Without rotation, the two tyre cavity resonances appear as vertical and horizontal modes (degenerate) however, when angular rotation is included, the two modes are offset from the vertical axis by a small angle, as illustrated in Feng et al. [19].

With an increase in axle force, the tyre contact results in a longer contact patch whose dimensions are obtained through trigonometric calculations. When an axle load is applied, the tyre cavity is compressed, while the tyre width increases to conserve volume, see Fig:7(a) for an illustration. The change in 
(b)

$(a)$

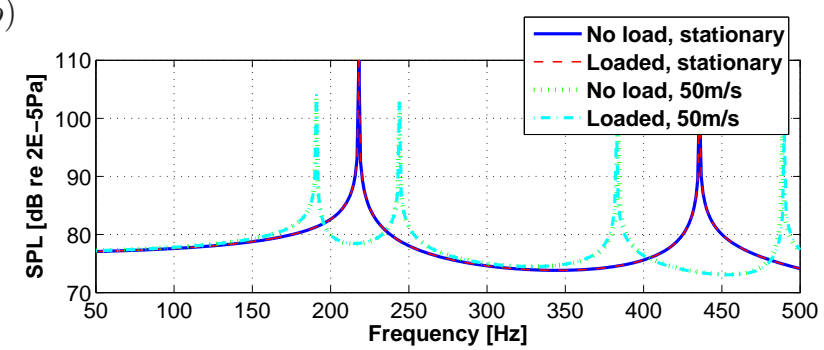

Figure 7: Impact of tyre loading: (a)Deformation of the tread and compression of the cavity, with an almost insignificant reduction in effective radius; (b) Change in tyre cavity frequency for a non-rotating wheel and a vehicle moving at $50 \mathrm{~m} / \mathrm{s}$ (colour online).

effective radius can be represented by significantly increasing the number of sections in the waveguide model (the grey lines) with only a low rate of change of cross sectional area between sections. Also, with vertical load the circumference is reduced (rolling radius), approximated by Pacejka's load tables (pp377 [21]), for example a $2 \mathrm{kN}$ load on a radial steel belt yields a $15 \mathrm{~mm}$ reduction in rolling radius which can be shown to have a minor effect on the cavity resonance (see the unloaded and loaded cases in Fig. 7 (b) where the vertical mode is presented).

The change in tyre cavity resonance frequency for the non-rotating case, is less than $10 \mathrm{~Hz}$ (from $211 \mathrm{~Hz}$ when unloaded to $218 \mathrm{~Hz}$ when the load is applied), which agrees very well with the literature [3, 4, 9, 18].

\section{Incorporation of passive resonators into the waveguide model}

The reduction in sound pressure inside the tyre cavity can be obtained through the use of one or more of two passive resonator designs, a quarterwavelength tube and a Helmholtz resonator. The former can be represented as a straight pipe of length $L_{q}$ and cross-section area $A_{q}$ with speed of sound $c_{q}$ applied to the junction between two sections as shown in Fig. 8 
(a)

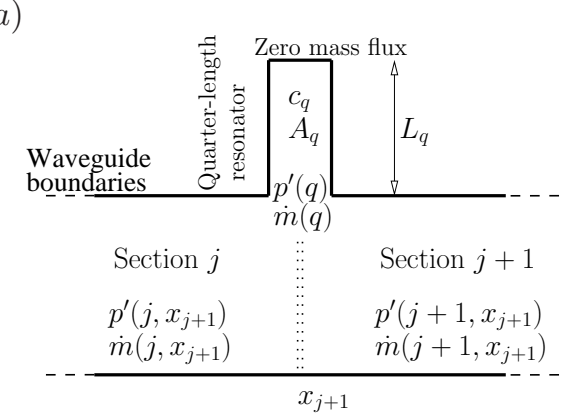

(b)

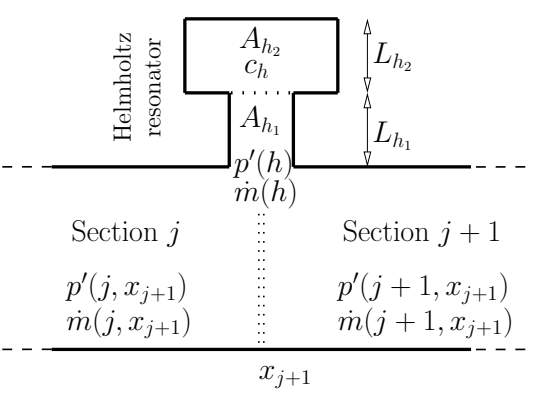

Figure 8: Passive resonators at a junction between two sections: (a) Quarter wavelength and (b) Helmholtz resonator.

The use of passive resonators in duct noise problems is widespread, however they have not found mainstream use in tyre cavity noise reduction as the dominant tyre cavity resonance frequency changes as the rotational speed changes 20]. Fernandez shows quarter wavelength tubes and implies that although the cavity resonance frequency disappears, two additional resonance of higher amplitude appear as replacements, negating the advantages [20].

In commercial wheel and tyre designs, the resonator can be placed either within the cavity itself or through designing a void in one of the wheel spokes, which are often hollow for weight saving, therefore their use as a resonator only requires a hole for the resonator orifice to be created next to the cavity. A quarter-length resonator can be represented as a length $L_{q}$ and cross-section area $A_{q}$ with one closed end resulting in total reflection of an incident wave due to the imposed boundary condition of zero mass flux.

The pressure in waveguide section $j$ at the boundary location $x_{j+1}$ is $p^{\prime}\left(j, x_{j+1}\right)$ and the pressure in section $j+1$ at the boundary location $x_{j+1}$ is $p^{\prime}\left(j+1, x_{j+1}\right)$.

At the junction shown in Fig. 8 (a), the pressure at the base of the resonator $p^{\prime}(q)$ is continuous, thus $p^{\prime}\left(j, x_{j+1}\right)=p^{\prime}\left(j+1, x_{j+1}\right)=p^{\prime}(q)$. The mass flux 
across the junction must also be consistent, thus $\dot{m}\left(j, x_{j+1}\right)=\dot{m}(q)+\dot{m}(j+$ $\left.1, x_{j+1}\right)$, so that

$$
\begin{aligned}
\dot{m}\left(j, x_{j+1}\right)= & \frac{A_{q}}{c_{q}} \frac{\left(1-e^{-2 \mathrm{i} \omega L_{q} / c_{q}}\right)}{\left(1+e^{-2 \mathrm{i} \omega L_{q} / c_{q}}\right)} p^{\prime}\left(j+1, x_{j+1}\right) \\
& +\dot{m}\left(j+1, x_{j+1}\right) .
\end{aligned}
$$

This allows a matrix equation to be written between the pressure perturbation and mass flux in one section of the waveguide $\mathbf{Y}_{j, x_{j}+1}$ and the adjacent section $\mathbf{Y}_{j+1, x_{j}}$, incorporating a quarter-wavelength resonator in the middle as

$$
\mathbf{Y}_{j, x_{j}+1}=\left[\begin{array}{cc}
1 & 0 \\
\left\{R+\frac{c_{q}}{A_{q}} \frac{\left(1+e^{-2 \mathrm{i} \omega L_{q} / c_{q}}\right)}{\left(1-e^{-2 \mathrm{i} \omega L_{q} / c_{q}}\right)}\right\}^{-1} & 1
\end{array}\right] \mathbf{Y}_{j+1, x_{j}}
$$

where the term in the brackets represents a transfer function between one section of the waveguide and another and $R$ is the rolling radius.

A similar process can be undertaken with respect to a Helmholtz resonator, however, the mechanism for sound reduction is very different. The quarterwavelength tube is designed to produce a reflected wave that cancels a harmonic incident wave while the Helmholtz resonator only produces a change in impedance which causes the incident wave to be reflected back towards source. As the cavity is periodic, this by itself will not be useful, however, the interaction of the gas flow with the neck dimensions creates thermo-acoustic losses where a small proportion of the incident energy is converted to waste heat.

This is one of the major problems with using multiple Helmholtz resonators, in that the single dominant frequency is split and in doing so, the broad band noise floor is raised. However, this noise floor is still substantially lower than the peak amplitude and traditional damping is more efficient at dealing with broad band noise rather than narrow band.

The body of the Helmholtz resonator, shown in Fig. 8(b), has length and cross-section area $L_{h_{2}}$ and $A_{h_{2}}$ respectively, with neck length and neck crosssection area $L_{h_{1}}$ and $A_{h_{1}}$, respectively. The speed of sound $c_{h}$. The pressure inside the volume of the resonator $p_{h_{2}}^{\prime}$ can be related to the mass flow $\dot{m}_{h}$ using the formulation by Dowling and Ffowcs Williams [7], $p_{h_{2}}^{\prime}=c_{h}^{2} \dot{m}_{h} /\left(\mathrm{i} \omega A_{h_{2}} L_{h_{2}}\right)$. The mass flow in the neck of the resonator can be related to the pressure change 
across the neck, $p_{h}^{\prime}-p_{h_{2}}^{\prime}=\mathrm{i} \omega L_{h_{1}} \dot{m}_{h} / A_{h_{1}}$. Through elimination of the pressure inside the volume terms, the pressure perturbation and mass flux at the neck of the resonator can be related by

$$
p_{h_{1}}^{\prime}=\left(\frac{\mathrm{i} \omega L_{h_{1}}}{A_{h_{1}}}+\frac{c_{h}^{2}}{\mathrm{i} \omega A_{h_{2}} L_{h_{2}}}\right) \dot{m}
$$

The process of incorporating the Helmholtz resonator into a transfer function between one section of the waveguide and another is then similar to the process previously shown for the quarter-wavelength resonator. Thus

$$
\mathbf{Y}_{j, x_{j}+1}=\left[\begin{array}{cc}
1 & 0 \\
\left(R+\frac{\mathrm{i} \omega L_{h_{1}}}{A_{h_{1}}}+\frac{c_{h}^{2}}{\mathrm{i} \omega A_{h_{2}} L_{h_{2}}}\right)^{-1} & 1
\end{array}\right] \mathbf{Y}_{j+1, x_{j}}
$$

The Helmholtz resonator produces a high impedance to an incoming wave when resonant conditions are imposed, this is the case when the right hand side of Eq. 10 is zero, which occurs at a frequency of $\omega_{h}=\sqrt{A_{h_{1}} c_{h}^{2} /\left(L_{h_{1}} L_{h_{2}} A_{h_{2}}\right)}$. In order that a singularity does not occur in the equations at their resonant frequencies, a resistance term $R$ is included representing losses in the fluid [22], see Eqs. 9111, where it is assumed that the fluid velocity moves with the tyre i.e. the fluid velocity with respect to the resonators is zero, thus losses associated with grazing flows are neglected. These can contribute significantly to the amplitude of the resistance and this assumption should be tested experimentally. Alternative formulations representing the attenuation loss in the pipe, viscous loss and radiation edge effects at the neck of the resonator are available from Bies and Hansen [23], however these give broadly similar amplitudes and frequency responses. Thus, the resistance in the Helmholtz resonator is

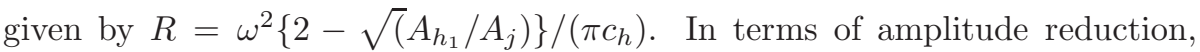
a smaller neck area is better, however, the machining tolerance then becomes more important, as small changes to the neck radius lead to larger changes in the tuned resonant frequency (see the next section for quantification of this effect). Finally, the effective neck length of the Helmholtz resonator $L_{h_{1}^{\prime}}$ is slightly longer than the physical neck length $L_{h_{1}}$ due to the additional virtual mass, therefore the corrected length suggested by Mechel [22] is incorporated, $\left.\left.{ }_{350} L_{h_{1}^{\prime}}=L_{h_{1}}+0.85 \sqrt{(} A_{h_{1}} / \pi\right)\left(2-\sqrt{(} A_{h_{1}} / A_{j}\right)\right)$. 
The implementation of losses in this paper provides for a generic design, however, once the initial design is finalised, it is important to either simulate (BEM/FEM) or experimentally test the final design to obtain highly accurate loss factors for the exact geometry of the neck, tapering, any champers, bevels

on edges and the exact shape of the neck and volume which are likely to be imposed through manufacturing constraints.

\subsection{Sensitivity requirements of resonator design}

Due to the low loss factor of air and the unrestricted cavity volume, the resonant frequencies of the two dominant cavity modes are very sharp, in contrast to the structural modes of the tyre, which has a loss factor dominated by the tyre tread rubber, see figures in [3]. Thus, any resonators addressing these cavity modes need to be carefully tuned in order to maximise attenuation, however, as the location of the resonant cavity frequencies change with tyre loading, vehicle speed and temperature.

The cavity mode is only a problem once the vehicle reaches a minimum speed, leading to two distinct cavity frequencies ( $f_{\text {lower }}$ and $\left.f_{\text {upper }}\right)$. Increasing the vehicle speed reduces $f_{\text {lower }}$ but increases $f_{\text {upper }}$, due to the Doppler effect. However, an increase in the cavity temperature leads to an increase in both $f_{\text {lower }}$ and $f_{\text {upper. }}$. As the lower frequency moves in opposite directions with respect to speed or temperature, so this should be the frequency tuned by any Helmholtz resonator, in order to maximise the efficiency if the coupled cavity resonance and structural design path is sensitive to a change in vehicle speed. Additionally, higher frequencies are more easily attenuated by traditional broad band damping treatments.

375 Introducing a single resonator into the wheel cavity only results in the mode rotating by ninety degrees so that the resonator is located at a nodal point. Similarly with two resonators located directly opposite each other, hence the effects discussed thus far require three resonators as a minimum. The inclusion of three Helmholtz resonators tuned to one of the frequencies of the cavity modes causes a reduction in amplitude at the critical frequency but also the generation of two further waves with lower amplitudes (corresponding to the wavelengths between resonators and contact patch) with a slightly higher and 


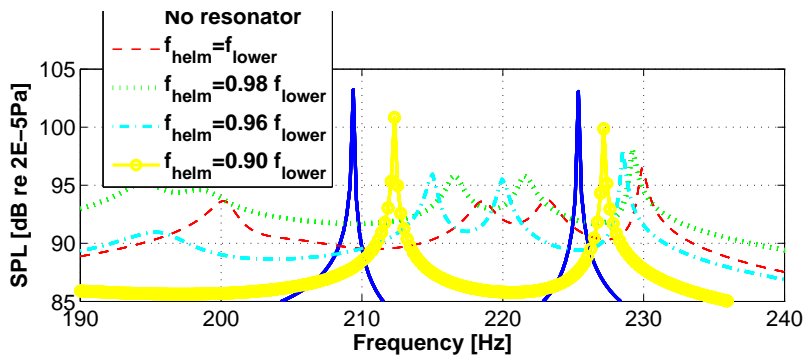

Figure 9: The effect of three Helmholtz resonators in a loaded wheel rotating at a vehicle speed of $54 \mathrm{~km} / \mathrm{hr}$, each tuned to a proportion of the lower tyre cavity resonant frequency (colour online).

lower frequency (hence all resonators tuned to the same value isn't necessarily useful).

For example, for a loaded tyre on a vehicle travelling at $54 \mathrm{~km} / \mathrm{hr}$, the two fundamental cavity frequencies are $f_{\text {lower }}=209.4 \mathrm{~Hz}$ and $f_{\text {upper }}=225.3 \mathrm{~Hz}$. Assume that three periodically spaced Helmholtz resonators are included in the tyre cavity, tuned to a frequency $f_{\text {helm }}$ that is proportional to the lower cavity resonance frequency $f_{\text {lower }}$. It can be seen in Fig. 9 that when $f_{\text {helm }}=f_{\text {lower }}$ (red dotted line), that the amplitude at $f_{\text {lower }}$ is indeed reduced substantially. There is a localised increase in broad-band sound which decreases at frequencies away from the Helmholtz tuning frequency, however this is more easily attenuated with damping materials than the narrow band sources.

Assume that two objective functions are created for the frequency range $f_{\text {crit }}=\left(f_{\text {lower }}+f_{\text {upper }}\right) / 2$; and ii $)$ the peak sound pressure level $p_{\text {peak upper for }}$ frequencies above $f_{\text {crit }}$. Note that the peak sound pressure level corresponding to the lower cavity resonance frequency is reduced by more than the upper one, as it is closer to the tuned resonator, however, the higher value is given in Table 3 summarising the results. So, when three perfectly tuned resonators are introduced tuned to $f_{\text {lower }}$, the peak amplitude $p_{\text {peak lower }}$ corresponding to $f_{\text {lower }}$ decreases from $103 \mathrm{~dB}$ to $93 \mathrm{~dB}$, however the amplitude $p_{\text {peak upper }}$ corresponding to $f_{\text {upper }}$ only reduces to $96 \mathrm{~dB}$. Nevertheless, three properly tuned resonators are able to reduce the peak cavity resonance amplitude by between 7 and $10 \mathrm{~dB}$. These values are similar to the experimental validation case reported by O'Boy and Walsh 24] using a static annular rig. Fig. 9 also 


\begin{tabular}{l|c|c}
\hline \hline Configuration & SPL $p_{\text {peak lower }}$ & SPL $p_{\text {peak upper }}$ \\
\hline & $\mathrm{dB}$ re $2 \times 10^{-5} \mathrm{~Pa}$ & \\
\hline No resonators & 103.2 & 103.2 \\
$f_{\text {helm }}=1.00 f_{\text {lower }}$ & 93.5 & 96.4 \\
$f_{\text {helm }}=0.98 f_{\text {lower }}$ & 95.9 & 98.1 \\
$f_{\text {helm }}=0.96 f_{\text {lower }}$ & 95.9 & 98.0 \\
$f_{\text {helm }}=0.90 f_{\text {lower }}$ & 100.8 & 100.0 \\
\hline \hline
\end{tabular}

Table 3: Analysis of the effectiveness of three Helmholtz resonators tuned to slightly different proportions of the lower resonance frequency in the tyre cavity.

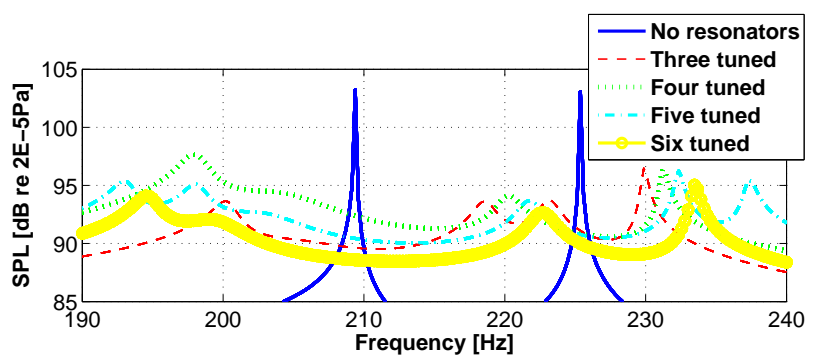

Figure 10: Sound pressure level against frequency in the tyre cavity when three, four, five and six Helmholtz resonators are included in the loaded wheel rotating at a vehicle speed of $54 \mathrm{~km} / \mathrm{hr}$, with each tuned to the lower tyre cavity resonant frequency (colour online).

shows results for Helmholtz resonators tuned to $0.98,0.96$ and 0.90 of the lower cavity resonance frequency $f_{\text {lower }}$. It can be seen that as the tuned resonator frequency becomes less well matched with the cavity resonance frequency that the tuned resonator becomes less effective. Note that the values in Table 3 are shown to 1 d.p. whereas in real life, the precision would be to round them to the nearest whole number. The above analysis shows a significant reduction in peak SPL amplitude when three resonators are perfectly tuned and also what happens when they are not (due to alterations in speed, temperature and machining tolerances).

It may also be possible to include more than three resonators with the design, and the change in sound pressure level inside the cavity of a loaded tyre rolling at $54 \mathrm{~km} / \mathrm{hr}$ with a different number of resonators, all tuned to $f_{\text {helm }}=f_{\text {lower }}$ is shown in Fig. 10. It can be seen that increasing the number of resonators isn't significantly helpful when they are all tuned to the same value.

A different strategy is to target both upper and lower cavity resonance frequencies simultaneously. Assume that several resonators are tuned to the lower 


\begin{tabular}{l|c|c}
\hline \hline Configuration & $\begin{array}{c}\text { SPL } \\
p_{\text {peak lower }}\end{array}$ & $\begin{array}{c}\text { SPL } \\
p_{\text {peak upper }}\end{array}$ \\
\hline & \multicolumn{2}{|c}{$\mathrm{dB}$ re $2 \times 10^{-5} \mathrm{~Pa}$} \\
\hline $\begin{array}{l}\text { No resonators } \\
5 \text { resonators }(3 \text { tuned to }\end{array}$ & 98.2 & 103.2 \\
$\begin{array}{l}\left.f_{\text {lower }}, 2 \text { tuned to } f_{\text {upper }}\right) \\
5 \text { resonators }((2 \text { tuned to }\end{array}$ & 95.9 & 96.7 \\
$\begin{array}{l}\left.f_{\text {lower }}, 3 \text { tuned to } f_{\text {upper }}\right) \\
\text { resonators equally spaced } \\
\text { between } f_{\text {lower }} \text { and } f_{\text {upper }}\end{array}$ & 97.4 & 95.1 \\
\hline \hline
\end{tabular}

Table 4: Sound pressure level in the tyre cavity rotating at $54 \mathrm{~km} / \mathrm{hr}$, with five resonators, in different tuned configurations.

frequency $f_{\text {helm }}=f_{\text {lower }}$ while several others are tuned to the upper frequency $f_{\text {helm }}=f_{\text {upper }}$. Table 4 illustrates the SPL reduction that can be achieved with the resonators. The resonators in the first, third and fourth circumferential positions are chosen for one setting and the second and fifth for the other. The result from five resonators tuned with equal frequency spacing is also shown. It is shown in Table 4 that this can be an effective method to reduce the amplitude of both resonance peaks simultaneously by approximately $7 \mathrm{~dB}$ (higher numerical precision is given in the table, but for actual use can be rounded).

An optimisation routine can be implemented to choose the correct frequency to tune the resonators for minimum average sound pressure. This will be bespoke to the requirements of the individual manufacturer (who might wish to avoid frequencies common to particular cabin resonances or structural transmission paths).

\subsection{Design optimisation}

The "optimum" resonator parameters can be determined using a generic multi-variable optimisation routine such as a Nelder-Mead Downhill Simplex method [25] with fixed limits to prevent non-physical parameters (such as negative lengths) 26]. The neck lengths of the resonators are adjusted to minimise the average peak sound pressure level in the cavity. The peak sound pressures are averaged over all angular positions in this case, rather than taking the peak sound pressure at a particular position in the cavity.

The results from an optimised resonator design on a tyre rotating between 


\begin{tabular}{l|c|c|c}
\hline \hline Configuration & SPL & SPL & SPL \\
& $p_{\text {peak lower }}$ & $p_{\text {peak upper }}$ & $p_{\text {average }}$ \\
\hline & \multicolumn{3}{|c}{$\mathrm{dB}$ re $2 \times 10^{-5} \mathrm{~Pa}$} \\
\hline No resonators & 103.2 & 102.7 & 103.0 \\
Three resonators & 88.4 & 87.5 & 87.9 \\
\hline \hline
\end{tabular}

Table 5: Peak sound pressure level averaged over rotational positions in the tyre cavity in a wheel rotating at between 54 and $108 \mathrm{~km} / \mathrm{hr}$, with three resonators, in different tuned configurations chosen by an optimisation routine. The critical frequency $f_{\text {lower }}$ ranges from 201.5 to $209.4 \mathrm{~Hz}$ while $f_{\text {upper }}$ ranges from 225.4 to $233.2 \mathrm{~Hz}$. Three resonators tuned to $f_{\text {helm } 1}=195.1 \mathrm{~Hz}, f_{\text {helm } 2}=215.4 \mathrm{~Hz}$ and $f_{\text {helm } 3}=229.6 \mathrm{~Hz}$.

\begin{tabular}{l|c|c|c}
\hline \hline Configuration & SPL & SPL & SPL \\
& $p_{\text {peak lower }}$ & $p_{\text {peak upper }}$ & $p_{\text {average }}$ \\
\hline & \multicolumn{3}{|c}{$\mathrm{dB}$ re $2 \times 10^{-5} \mathrm{~Pa}$} \\
\hline No resonators & 103.2 & 102.7 & 103.0 \\
Five resonators & 88.5 & 85.5 & 87.0 \\
\hline \hline
\end{tabular}

Table 6: Peak sound pressure level averaged over rotational positions in the tyre cavity rotating at between 54 and $108 \mathrm{~km} / \mathrm{hr}$, with five resonators, in different tuned configurations chosen by an optimisation routine. The critical frequency $f_{\text {lower }}$ ranges from 201.5 to $209.4 \mathrm{~Hz}$ while $f_{\text {upper }}$ ranges from 225.4 to $233.2 \mathrm{~Hz}$. Five resonators tuned to $f_{\text {helm } 1}=199.6 \mathrm{~Hz}$, $f_{\text {helm } 2}=210.0 \mathrm{~Hz}, f_{\text {helm } 3}=217.9 \mathrm{~Hz}, f_{\text {helm } 4}=224.8 \mathrm{~Hz}$ and $f_{\text {helm } 5}=236.6 \mathrm{~Hz}$.

54-108 km/hr are shown in Table 5 where three resonators with different tuning values are utilised. As can be seen, the reduction in peak sound pressure level is approximately $15 \mathrm{~dB}$. Also of interest is that the first Helmholtz tuning frequency $f_{\text {helm }}$ has a value lower than $f_{\text {lower, }}$, with the second tuning frequency $f_{\text {helm }}$ a value between $f_{\text {lower }}$ and $f_{\text {upper }}$.

The comparable optimisation over the same speed range for the case where five resonators are available is shown in Table 6. where it can be seen that the optimised design reduces the peak sound pressure level from $103 \mathrm{~dB}$ to $87 \mathrm{~dB}$ (a reduction of $16 \mathrm{~dB}$ ).

The optimisation routine provides a method to weight certain frequencies to avoid, or concentrate on, so that design of suspension components can be made without creating transmission paths.

A final consideration in the optimisation is to use the resonators to move the resulting cavity frequencies above the value for which interior NVH noise is concerning, thus away from the frequencies where the occupants will likely hear.

This methodology provides the fast initial design for the resonators, however it is then important to i) carry out experiments on the exact geometry of the 
resonators, to obtain exact resistance terms and ii) carry out detailed finite element / boundary element calculations on the coupled acoustic, structural tyre and suspension problem. Critically, this requires all detailed geometry 5 and material properties, of the frequency response functions of the tyre and suspension strut, which are often not available to automotive designers (being specific to the part manufacturers). On this basis, using an uncoupled design tool has distinct advantages prior to full scale experimental implementation.

\section{Conclusions}

In this paper, a design tool for the reduction of tyre cavity noise through the suppression of the fundamental resonance frequency using passive resonators has been developed. This tool is a fast uncoupled acoustic model which estimates the sound pressure level inside a tyre cavity which is rotating, while loaded at a contact patch and can incorporate a variety of gases. The passive resonators

475 have been tuned using engineering judgement and also an optimisation strategy to minimise the sound pressure level over a range of frequencies and operating conditions.

It has been shown that while the tyre cavity natural frequency is simple to calculate under static conditions, when realistic effects such as rotation, ground loading and temperature are included, the frequency changes significantly. This introduces considerable difficultly in choosing the best resonator design.

Using reasonable engineering guesses for the placement of the resonators with a fixed rotation speed provides a reduction of approximately $7 \mathrm{~dB}$, in terms of peak amplitude values (the narrow band amplitude decreases by approximately

$48513 \mathrm{~dB}$ ). When the speed of the tyre varies from $54-108 \mathrm{~km} / \mathrm{hr}$, by applying an optimisation routine for the resonator design, a reduction in the average peak amplitude of the sound pressure from $103 \mathrm{~dB}$ to $88 \mathrm{~dB}$ and from $103 \mathrm{~dB}$ to $87 \mathrm{~dB}$ corresponding to the placement of three or five optimised and individually tuned resonators has been achieved.

\section{Acknowledgements}

All data created during this research is available from the Loughborough University data archive at: 10.17028/rd.lboro.5017130 


\section{References}

[1] D. R. Raichel, Noise, vibration and harshness (NVH) criteria as functions

495 10
[4] J. K. Thompson, Plane wave resonance in the tire air cavity as a vehicle interior noise source, Tire Science and Technology 23 (1) (1995) 2 - 10.

[5] R. W. Scavuzzo, L. T. Charek, P. M. Sandy, P. M. Shteinhauz, Influence of wheel resonance on tire acoustic cavity noise, SAE Technical Paper 940533.

[6] L. R. Molisani, R. A. Burdisso, D. Tsihlas, A coupled tire structure/acoustical cavity model, International Journal of Solids and Structure 40 (1) (2003) 5125-5138.

[7] A. P. Dowling, J. E. Ffowcs Williams, Sound and Sources of Sound, Ellis Horwood, 1989.

[8] W. A. Leasure, E. K. Bender, Tire-road interaction noise, Journal of the Acoustical Society of America 58 (1) (1975) 39-50.

[9] R. Gunda, S. Gau, C. Dohrmann, Analytical model of tire cavity resonance and coupled tire/cavity modal model, Tire Science and Technology 28 (1) (2000) 33-49.

[10] D. C. Gazis, Three dimensional investigation of the propagation of waves in hollow circular cylinders- i. analytical foundation ii. numerical results, Journal of the Acoustical Society of America 31 (1959) 568-78.

[11] D. J. O'Boy, A. P. Dowling, Tyre / road interaction noise: A 3D viscoelastic multilayer model of a tyre belt., Journal of Sound and Vibration 322 (2009) 829-850. 
[12] G. Kim, Flexural wave propagation and sound radiation of the tyre shell, in: The 2001 Congress and Exposition on Noise Control Engineering, 27-30 August, The Hague, Holland, 2001.

[13] G. Kim, D. Lee, Y. K. Kang, Influence of design factors on tyre acoustic cavity noise, in: The 2005 Congress and Exposition on Noise Control Engineering, 7-10 August, Rio de Janerio, Brazil, 2005.

[14] Z. Feng, P. Gu, Modeling and experimental verification of vibration and noise caused by the cavity modes of a rolling tire under static loading, SAE Technical Paper 2011-01-1581.

[15] A. Bennetts, N. S. Crookes, Tyre noise measurement - a new approach (tyre cavity microphone - TCM), Engineering Integrity 33 (2012) 16-20.

[16] J. J. Lee, H. Q. Pham, J. A. Moore, Structure borne vibration transmission in a tire and wheel assembly, Tire Science and Technology 26 (3) (1998) $173-185$.

[17] A. Landfester, D. Giljohann, Finite/infinite element methods for the helmholtz equation in large exterior domains including damping and flow, in: Proceedings of the International Conference on Sound and Vibration, ICSV17, Cairo, Egypt, 2000, pp. 2021-2028.

[18] P. Kindt, F. De Coninck, P. Sas, W. Desmet, Analysis of tire/road noise caused by road impact excitations, in: Proceedings of the International Styrian Noise, Vibration and Harshness Congress. International Styrian Noise, Vibration \& Harshness Congress, 4-6 June, Graz, Austria, 2008, pp. $113-134$.

[19] Z. C. Feng, P. Gu, Y. Chen, Z. Li, Modeling and experimental investigation of tire cavity noise generation mechanisms for a rolling tire, in: SAE 2009 Noise and Vibration Conference and Exhibition, St. Charles, IL, USA, 2009.

[20] E. T. Fernàndez, The influence of tyre air cavities on vehicle acoustics, Ph.D. thesis, Department of Aeronautical and Vehicle Engineering, Kungliga Tekniska Högskolan (2006). 
550 [21] H. Pacejka, Tyre and Vehicle Dynamics, Automotive engineering, Butterworth-Heinemann, 2006.

[22] F. P. Mechel, Formulas of acoustics, Springer Science \& Business Media, 2013.

[23] D. A. Bies, C. H. Hansen, Engineering Noise Control: Theory and practice, $555 \quad$ Spon, 2003.

[24] D. J. O'Boy, S. J. Walsh, Automotive tyre cavity noise modelling and reduction, in: Proceedings of Internoise, 2016.

[25] J. A. Nelder, R. Mead, A simplex method for function minimisation, The Computer Journal 7 (1965) 308-313.

${ }_{560}$ [26] D. J. O'Boy, A. P. Dowling, Tyre / road interaction noise: Numerical noise prediction of a patterned tyre on a rough road surface., Journal of Sound and Vibration 323 (2009) 270-291. 